\title{
Statistical issues and approaches in endophenotype research
}

\author{
SHAM Pak Chung $^{1 *}$, CHERNY Stacey $\mathrm{S}^{1} \&$ HALL Mei-Hua ${ }^{2}$ \\ ${ }^{1}$ Department of Psychiatry and The State Key Laboratory of Brain and Cognitive Sciences, The University of Hong Kong, Hong Kong, China; \\ ${ }^{2}$ Psychology Research Laboratory, McLean Hospital, Harvard Medical School, Boston, MA 02115-6092, USA
}

Received July 28, 2011; accepted August 8, 2011; published online September 12, 2011

\begin{abstract}
The endophenotype concept was initially proposed to enhance the power of genetic studies of complex disorders. It is closely related to the genetic component in a liability-threshold model; a perfect endophenotype should have a correlation of 1 with the genetic component of the liability to disease. In reality, a putative endophenotype is unlikely to be a perfect representation of the genetic component of disease liability. The magnitude of the correlation between a putative endophenotype and the genetic component of disease liability can be estimated by fitting multivariate genetic models to twin data. A number of statistical methods have been developed for incorporating endophenotypes in genetic linkage and association analyses with the aim of improving statistical power. The most recent of such methods can handle multiple endophenotypes simultaneously for the greatest increase in power. In addition to increasing statistical power, endophenotype research plays an important role in helping to understand the mechanisms which connect the associated genetic variants with disease occurrence. Novel statistical approaches may be required for the analysis of the complex relationships between endophenotypes at different levels and how they converge to cause the occurrence of disease.
\end{abstract}

psychiatric disorders, endophenotypes, genetics, genomics, association, linkage

Citation: Sham P C, Cherny S S, Hall M H. Statistical issues and approaches in endophenotype research. Chinese Sci Bull, 2011, 56: 3403-3408, doi: 10.1007/ s11434- 011-4746-y

Most common human diseases, such as coronary heart disease, diabetes, cancers, bipolar affective disorders and schizophrenia, have complex aetiology. While they tend to cluster in families, they do not exhibit the characteristic Mendelian segregation ratios of single-gene disorders. These diseases therefore cannot be solely caused by a single genetic mutation, but have a more complex genetic architecture. Over the last 3 decades, various genetic approaches, starting with linkage scans, followed by candidate gene and targeted association studies, and most recently genomewide association studies (GWAS), have been used to identify and characterize the genetic loci for such complex diseases. While many susceptibility loci have been identified, the vast majority of these loci have very small effects on disease risk, typically with odds ratios under 1.5. Even in aggregate, the loci so far identified fall far short of being

*Corresponding author (email: pcsham@hku.hk) able to explain the level of familial clustering observed for the diseases. This appears to be especially the case for psychiatric disorders such as schizophrenia, bipolar affective disorder and unipolar depression.

The endophenotype strategy has been proposed as a way to improve the statistical power of genetic studies (e.g., [1]). An endophenotype is a quantitative phenotype which measures some neurobiological mechanisms in the pathways from genotype to phenotype. The criteria for an endophenotype include four elements: (a) the presence of a correlation between the disease and putative endophenotype in the same individual, (b) evidence that the putative endophenotype precedes the disease and is therefore likely to be a cause rather than a consequence of the disease, (c) evidence that the correlation between the disease and the putative endophenotype occurs at the familial as well as the individual level, and (d) evidence that this familial correlation is at least partially explained by genes that have an influence 
on both disease risk and the putative endophenotype.

Although the concept of endophenotype was introduced for psychiatric disorders (e.g., [2]), it is also applicable to medical conditions. For instance, blood pressure and blood sugar levels can be considered as endophenotypes for hypertension and diabetes, respectively.

\section{Statistical properties of a perfect endophenotype}

Under the liability-threshold model, susceptibility to disease is a normally distributed variable in the population, but is not directly measured. Under this model, individuals whose liability exceeds a certain threshold value develop the disease. For multifactorial diseases, heritability refers to the proportion of variance in liability explained by genetic factors. Heritability estimation therefore explicitly assumes a liability-threshold model.

Under a liability-threshold model, the perfect endophenotype for a disease is a trait that is perfectly correlated to the genetic component of liability. Assuming both the genetic and environmental components of liability to be normally distributed and independent, a perfect endophenotype should also be normally distributed, and have an average deviation from the population mean (in standard deviation units) in affected individuals of

$$
D=\frac{h \rho\left(\Phi^{-1}(K)\right)}{K}
$$

where $K$ is the population frequency of the disease, $h$ is the square root of the heritability of the disease, $\rho$ is the standard normal density function and $\Phi^{-1}$ is the inverse standard normal distribution function. For first-degree relatives of affected individuals, the expected deviation of the endophenotype from the population mean is $D / 2$ standard deviation units. For example, for a disease with population frequency of $1 \%$ and heritability of 0.8 , the average deviation of a perfect endophenotype would be 2.38 standard deviation units, while for first-degree relatives would be 1.19 standard deviation units.

Having a perfect endophenotype can lead to substantial power increases in genetic association studies. For example, in a simple case-control study, for a disease with $K=0.01$, a risk allele with frequency 0.3 and allelic odds ratio 1.2 acting in a multiplicative fashion, the required sample size for $80 \%$ power is 5376 cases and equal number of controls, for the genome-wide significance level of $5 \times 10^{-8}$ (Genetic Power Calculator [3], http://pngu.mgh.harvard.edu/ purcell/ gpc/qcc.html). If, however, the controls are selected to be below the population average in endophenotype value, then the sample size required will be reduced to 3265 cases and 3265 controls. If the controls are selected to be from the opposite extreme $1 \%$ of the endophenotype distribution, then the required sample size will be reduced further to 1414 cases and 1414 controls.

\section{Modelling disease-endophenotype relationships}

The classical twin design is one of the most powerful study designs for estimating the relative contribution of genetic and environmental factors to human traits or diseases [4,5]. The classical twin study consists of a design in which data are collected from identical or monozygotic (MZ) twins and fraternal or dizygotic (DZ) twins reared together in the same environment. Based on quantitative genetic theory, a phenotype (an observed trait) can be partly a result of genetic factors $(G)$ and partly influenced by environmental factors. There are two sources of environmental variances: common environmental variance $(C)$ and individual-specific environmental variance $(E) . C$ is due to the shared environment within the family members. E results from influences that are unique to a person, and often also includes measurement error. The total phenotypic variance of a trait is the sum of all three sources of variances, $G+C+E$ [6]. If we assume that the effect of shared environment impacts $\mathrm{MZ}$ and DZ twin pairs equally, then the difference in covariance between $\mathrm{MZ}$ twins, who share all genes, and DZ twins, who share half their genes on average, indicates the extent to which genetic factors influence the trait. Heritability $\left(h^{2}\right)$ is the proportion of the total phenotypic variance explained by genetic factors [6].

When multiple traits (such as a disease and a putative endophenotype) are collected in the same MZ and DZ twin pairs, it is possible not only to estimate the heritability of each trait but also to address questions such as whether the same set of genes accounts for variation in more than one phenotype. And if so, to what extent do the same genes explain the covariation between the phenotypes?

In the multivariate twin analysis, the focus is on the covariance between traits across twins rather than the variance of each trait considered separately [7]. Consider two phenotypes, schizophrenia and sensory gating event related potential (ERP), for example. MZ and DZ correlations are compared across the two traits; that is, twin one's affection status for schizophrenia is correlated with the co-twin's sensory gating ERP score. If the overall cross-twin crosstrait correlations are greater for $\mathrm{MZ}$ than for $\mathrm{DZ}$ twins, this suggests a genetic overlap between the two traits. If the cross-twin cross-trait correlations are the same and greater than zero for $\mathrm{MZ}$ and $\mathrm{DZ}$ pairs, it indicates only shared common environmental $(C)$ influences contributing to the correlation. If the cross-twin cross-trait correlations are non-significant for both $\mathrm{MZ}$ and $\mathrm{DZ}$ pairs, but there is a significant phenotypic correlation between schizophrenia and sensory gating ERP score, it indicates the effects of specific environment $(E)$ on the observed phenotypic correlation. 


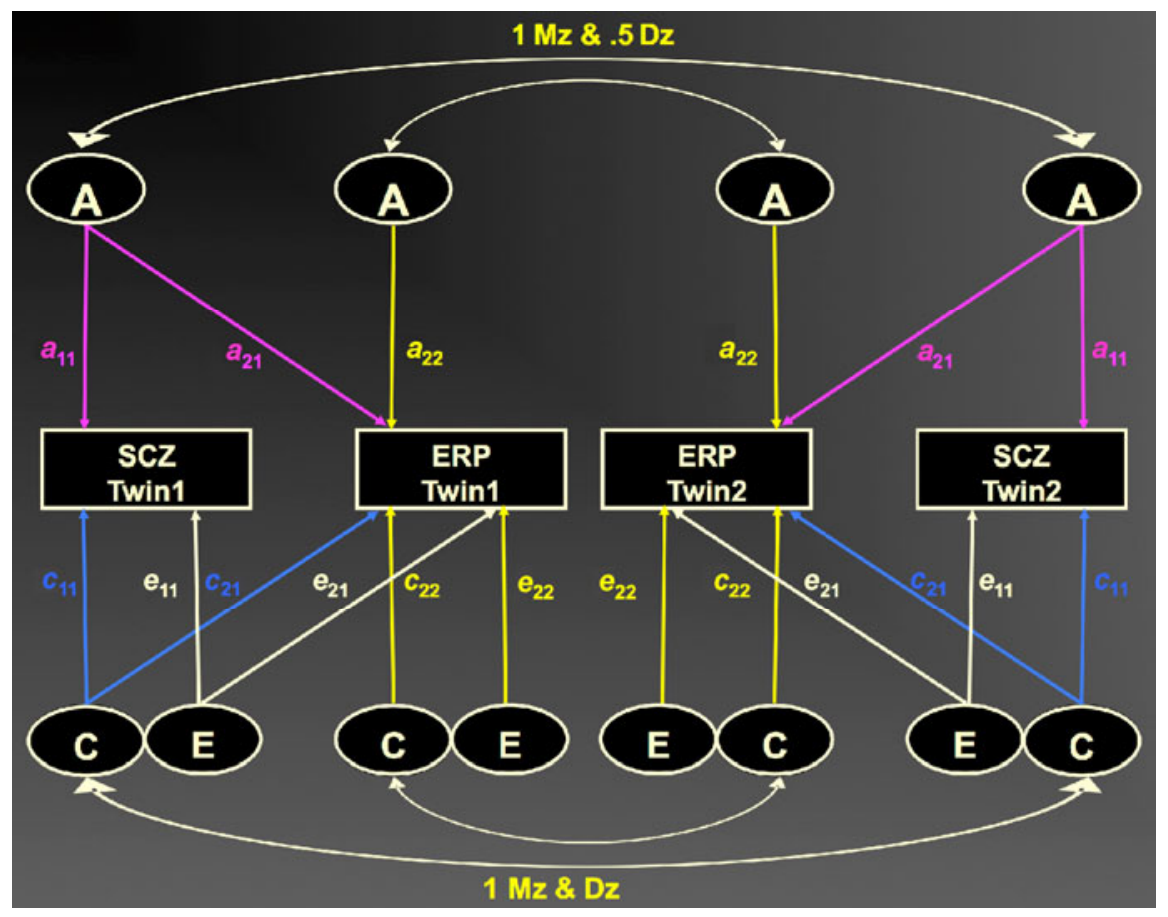

Figure 1 Modelling disease-endophenotype relationships — path diagram for bivariate model.

The genetic correlation $\left(R_{g}\right)$ for a bivariate model, as illustrated in Figure 1, is derived as the genetic covariance between trait 1 (i.e., schizophrenia) and 2 (i.e., sensory gating ERP), given as $a_{11} a_{21}$, divided by the square root of the product of the genetic variances of trait $1\left(a_{11}{ }^{2}\right)$ and $2\left(a_{21}{ }^{2}+\right.$ $a_{22}^{2}$ ):

$$
R_{g}=\frac{a_{11} a_{21}}{\sqrt{a_{11}^{2} \times\left(a_{21}^{2}+a_{22}^{2}\right)}} .
$$

Similarly, the common $\left(R_{c}\right)$ and specific $\left(R_{e}\right)$ environmental correlations between schizophrenia and sensory gating ERP are derived as the $C$ and $E$ covariances between trait 1 and 2 divided by the square root of the products of the $C$ and $E$ variances of trait 1 and 2, respectively:

$$
\begin{aligned}
& R_{c}=\frac{c_{11} c_{21}}{\sqrt{c_{11}^{2} \times\left(c_{21}^{2}+c_{22}^{2}\right)}} . \\
& R_{e}=\frac{e_{11} e_{21}}{\sqrt{e_{11}^{2} \times\left(e_{21}^{2}+e_{22}^{2}\right)}} .
\end{aligned}
$$

The phenotypic correlation, $\boldsymbol{R}_{\boldsymbol{p} \boldsymbol{h}}$, is the sum of the product of the genetic correlation and the heritabilities of the two phenotypes, the product of the $c$ correlation and the $c$ effects of the two phenotypes, and the product of the $e$ correlation and the $e$ effects of the two phenotypes:

$$
\begin{aligned}
& R_{\mathrm{ph}}=\sqrt{h_{\text {trait1 }}^{2}} \times R_{g} \times \sqrt{h_{\text {trait } 2}^{2}}+\sqrt{c_{\text {trait1 }}^{2}} \\
& \times R_{c} \times \sqrt{c_{\text {trait } 2}^{2}}+\sqrt{e_{\text {trait1 }}^{2}} \times R_{e} \times \sqrt{e_{\text {trait2 }}^{2} .}
\end{aligned}
$$

If the genetic correlation is 1 , schizophrenia and sensory gating ERP share completely overlapping genetic influences. If the genetic correlation is less than 1 , at least some genes are specific to only one trait. A large genetic correlation, however, does not imply a large phenotypic correlation, as the latter is also a function of the heritabilities. If these are low, the genetic contribution to the observed correlation will also be low.

The twin design, when augmented by sophisticated structural equation modeling (SEM) techniques and multivariate analyses, thus allows for the simultaneous modeling of diseases and endophenotypes to determine their common genetic aetiology. The power to detect disease risk genes will only be increased through the use of endophenotypes if their association is due to pleiotropic genetic effects [8].

When studying rare diseases such as schizophrenia (SCZ), it is often a more efficient strategy to collect data from selected samples (e.g., through affected probands). Modelling disease-endophenotype relationships in selected samples requires an ascertainment correction. This means that the fit function used in the SEM has to be corrected for the proportion of the population that is not observed. One method for correcting incomplete ascertainment is by introducing an ascertainment probability for each cell (values between 0 and 1) and multiplying the cell probabilities with these ascertainment probabilities in a user defined fitfunction [9]. An alternative way is by fixing the model parameters for the selection variable (i.e., disease status) [10].

An example of modeling disease-endophenotype relationship can be found in the Maudsley Schizophrenia Twin 
Study [11]. This study involved a sample of concordant MZ SCZ twins, discordant MZ SCZ twins, and healthy MZ and DZ twin pairs. Data on three putative neurophysiological endophenotypes were collected (Sensory gating ERP, P300 ERPs, and mismatch negativity [MMN]). SEM analyses were applied to: (a) estimate the heritability of each ERP index, (b) quantify the relationship between each ERP phenotype and SCZ, and (c) test the significance of partitioned genetic and environmental correlations with the illness. The results indicated that all three ERP phenotypes are heritable traits. Also, significant phenotypic correlations were found between SCZ and each ERP phenotype; i.e., SCZ was significantly associated with smaller P300 amplitude $\left(r_{p h}=\right.$ $-0.35 ; 95 \% \mathrm{CI}=-0.50$ to -0.19$)$, decreased sensory gating $\left(r_{p h}=0.40 ; 95 \% \mathrm{CI}=0.24\right.$ to 0.53$)$, and reduced MMN $\left(r_{p h}\right.$ $=0.31 ; 95 \% \mathrm{CI}=0.15$ to 0.46$)$. Further, the main source of the observed phenotypic association was due to significant genetic correlations. Sensory gating had the highest genetic correlations with SCZ $\left(R_{g}=0.59\right)$, followed by the P300 amplitude $\left(R_{g}=-0.48\right)$, suggesting that these measures are valid SCZ endophenotypes. MMN, on the other hand, had the lowest genetic correlation $\left(R_{g}=0.31\right)$ [11].

\section{Incorporating endophenotypes into linkage and association analyses to increase power}

Typical linkage studies of complex disease have generally relied on the use of affected sibling pairs. The methodology relies on the measurement of allelic sharing identical by descent (ibd). On average, siblings share $50 \%$ of their chromosomal material ibd. If a disease-causing locus is present, sharing will increase above $50 \%$ at that particular location in the genome. Conversely, a pair of sibs who are discordant for the disease will share less than $50 \%$ at a disease locus. If a common disease is present in $10 \%$ of the population, under the liability threshold model, we know that those affected score somewhere in the top $10 \%$ of the liability distribution. Those who are not affected score somewhere in the bottom $90 \%$ of this liability distribution. Given that, it is obvious that affected sibling pairs could be highly informative, as both score in the extreme of the liability distribution. However, a discordant pair is a lot less informative, since the unaffected member can score almost anywhere on the unmeasured liability (the bottom 90\%).

However, the use of an endophenotype which is a quantitative trait and highly correlated with disease status allows a much more precise estimate of both an affected and an unaffected individual's liability score. If we have a proxy for liability in the quantitative endophenotype, instead of ascertaining a sample of simply concordant and discordant sibling pairs, we can select for extreme concordance and extreme discordance. Instead of selecting individuals in the bottom $90 \%$ of the liability distribution, for example, we can consider only those who score in the bottom $10 \%$ as unaffected. Furthermore, having available meaningful quantitative data on both cases and controls, and using analysis methods which make use of quantitative data, further increases power over using simple affection status.

An example of the use of endophenotypes in linkage analysis to ascertain sib pairs who are both extremely concordant and extremely discordant is the large population-based sample of sibships assessed for anxiety and depression measures in the GENESiS study [12]. This study involved collection of questionnaire data on a large community-based sample of 34,371 people, of which 14,807 were members of sibships of sizes $2-7$, with the majority sib pairs. These questionnaires comprised several wellvalidated instruments for assessing anxiety and depression symptoms, personality traits, and psychological distress. Using an algorithm implemented in the SEL (SELection for QTL Linkage) program [13], the goal was to obtain DNA samples from the most informative $10 \%$ of all sibships, based on their composite phenotypic scores across four questionnaire-based assessments. Informativeness was then recalculated on the $65 \%$ of samples who returned DNA and another round of selection was performed to choose those to be genotyped on 400 microsatellite markers, for subsequent quantitative trait linkage analysis. Sibships which are most discordant or extreme concordant on the phenotype are the most informative, with their informativeness a function of the sibling correlation on the phenotype. The SEL program ranks the sibships based on their likely contribution to the noncentrality parameter for linkage, using only phenotypic information. Given that this was a selected sample, it was ideally suited for regression-based linkage analysis [14]. The study also employed an average of two repeated measures of the Eysenck personality questionnaire (EPQ-N; Eysenck \& Eysenck, 1975), measured on two occasions in $78 \%$ of those subjects genotyped, rather than just a single measurement occasion, reducing measurement error and thereby increasing heritability [6] and power. Merlinregress was extended to make use of this additional information, allowing the use of averaged phenotypes from any number of measurement occasions on a per-subject basis, with the method taking account of the differing expected error variance on a per-subject basis (see Appendix in [12]). This analysis resulted in a LOD of nearly 3 on Chromosome 6 for the EPQ-N. This study further refined the analysis by examining males and females separately, yielding a somewhat higher LOD score [12]. Through the use of endophenotypes, rather than simple binary measures of depressed vs. not depressed, power was increased by discriminating and selecting unaffected people as controls who, in fact, were the least neurotic, an endophenotype underlying depression. This is a superior approach to using controls who are simply not depressed, which would be a very heterogeneous group including people who range from borderline depressed to those lacking any neurotic tendencies completely.

In the past few years, the field of psychiatric genetics has 
moved completely away from genome-wide linkage studies to GWAS. However, the same advantages of the use of endophenotypes apply to GWAS. Thus far, there have been few GWAS employing endophenotypes (e.g., [15,16]), as primary analyses have been for diagnoses of diseases such as schizophrenia and bipolar disorder [17-20]. Nonetheless, we expect to see secondary analyses of endophenotypes in those studies which have collected them. Our present statistical genetic analysis toolset is ready for such analyses in GWAS, with software such as Merlin/QTDT [21-23] and PLINK [24] capable of handling quantitative traits, both in family designs and studies of unrelated individuals. Power of such secondary analyses may not be much greater than the original studies of the disorder phenotype, however, unless supernormal controls are included whose phenotypic scores are at the opposite extreme of the distribution than those of the cases.

\section{Model with multiple endophenotypes}

For many diseases, investigators routinely measure multiple endophenotypes on the same person in addition to clinical diagnosis. The strategy of modeling multiple endophenotypes in genetic linkage or association analyses can identify not only trait-specific quantitative trait loci (QTL), but also those shared between correlated traits. Studies have shown that including multiple endophenotypes that are correlated with each other in the genetic analyses can substantially increase power [25-28]. At the clinical level, studying multiple endophenotypes within a patient sample may help identify homogeneous subtypes across diagnostic categories by classifying patients based on similar functional, neurophysiological, or behavior profiles (e.g., deficits in frontal working memory, treatment prognosis, medication response). These profiles, in turn, can be used in statistical analyses to examine pleiotropic effects of a gene. Therefore, including multiple correlated phenotypes as well as selecting genetically homogenous phenotypes would increase the signal to noise ratio in linkage and association studies. An efficient multivariate test of association for populationbased designs has been implemented in PLINK [24].

\section{Characterization of differential impact of contributing variants}

The utility of the disease-endophenotype mapping approach can be extended beyond improving statistical power for gene identification [29]. An important feature of endophenotypes is that the underlying molecular and neurobiology can be systematically examined and characterized in humans and animals [30,31]. Such neurobiological insight becomes valuable in molecular genetic studies for dissecting the functional effects of risk genetic variants or for identifying biological mechanisms of a disease-that is, linking the pathway from genotype to phenotype [29,32]. For example, GWAS analyses have revealed that variations in the CACNAIC (alpha 1C subunit of the L-type voltage-gated calcium channel) gene are associated with susceptibility to bipolar disorder [BPD], SCZ and major depression [MDD] $[33,34]$. However, this gene was not previously a candidate and its role in the neurobiology and neurophysiology of BPD, SCZ, or MDD was unclear. Follow-up association studies using various endophenotypes found that the risk genotype predicts greater hippocampal activity during emotional processing, increased prefrontal activity during executive cognition, and increased expression of mRNA in human brain $[35,36]$. Also, an animal study found that $C A C N A 1 C$ haploinsufficiency modifies a number of anxiety and depressive-like behaviors and interacts with sex to influence behavior [37]. Such phenotypic dissection of associations found via GWAS will be necessary to gain a true understanding of the effect of all such newly identified loci.

\section{Conclusions}

The endophenotype concept was initially proposed to enhance the power of genetic studies of complex disorders. It is closely related to the genetic component in a liability-threshold model; a perfect endophenotype should have a correlation of 1 with the genetic component of the liability to disease. Whether a putative endophenotype is a perfect representation of the genetic component of liability to a disease can be gleaned from the average deviations of the endophenotype values among affected proband subjects and their first-degree relatives, in relation to the prevalence and heritability of the disease in the population. Selecting controls to be from the opposite extreme of the endophenotype distribution from the cases can substantially improve statistical power of genetic association studies.

In reality, a putative endophenotype is unlikely to be a perfect representation of the genetic component of disease liability. The magnitude of the correlation between a putative endophenotype and the genetic component of disease liability can be estimated by fitting multivariate genetic models to twin data. Twin modeling can be complicated by the ascertainment procedure of the twin sample, but there are appropriate statistical methods to allow for this.

A number of statistical methods have been developed for incorporating endophenotypes in genetic linkage and association analyses with the aim of improving statistical power. The most recent of such methods can handle multiple endophenotypes simultaneously for the greatest increase in power.

In addition to increasing statistical power, endophenotype research plays an important role in helping to understand the mechanisms which connect genetic variants with disease occurrence. The causal pathways are likely to be 
very complicated and involve endophenotypes at multiple levels: from RNA expression profiles and patterns of protein expression, through neuronal and synaptic properties, to neurophysiological and neurocognitive function. Novel statistical approaches may be required for the analysis of the complex relationships between endophenotypes at different levels, to understand how they converge to cause the occurrence of disease.

The work was supported by the Hong Kong Research Grants Council General Research Fund (HKU 766906M and HKU 774707M), the European Community's Seventh Framework Programme under grant agreement No. HEALTH-F2-2010-241909 (Project EU-GEI), the University of Hong Kong (HKU) Strategic Research Theme of Genomics, HKU Small Project Funding (201007176248), and the National Institute of Mental Health of the USA (1K01MH086714).

1 Almasy L, Blangero J. Endophenotypes as quantitative risk factors for psychiatric disease: Rationale and study design. Am J Med Genet, 2001, 105: 42-44

2 Gottesman I I, Gould T D. The endophenotype concept in psychiatry: Etymology and strategic intentions. Am J Psychiatry, 2003, 160: 636-645

3 Purcell S, Cherny S S, Sham P C. Genetic power calculator: Design of linkage and association genetic mapping studies of complex traits. Bioinformatics, 2003, 19: 149-150

4 Boomsma D, Busjahn A, Peltonen L. Classical twin studies and beyond. Nat Rev Genet, 2002, 3: 872-882

5 Martin N, Boomsma D, Machin G. A twin-pronged attack on complex traits. Nat Genet, 1997, 17: 387-392

6 Falconer D S, Mackay T F C. Introduction to Quantitative Genetics. Harlow: Longman, 1996. xv, 464

7 Neale M C, Cardon L R. North Atlantic Treaty Organization. Scientific Affairs Division. Methodology for Genetic Studies of Twins and Families. Dordrecht, Boston: Kluwer Academic Publishers, 1992. xxv, 496

8 Posthuma D, Beem A L, de Geus E J, et al. Theory and practice in quantitative genetics. Twin Res, 2003, 6: 361-376

9 Cardno A G, Rijsdijk F V, Sham P C, et al. A twin study of genetic relationships between psychotic symptoms. Am J Psychiatry, 2002, 159: 539-545

10 Rijsdijk F V, van Haren N E, Picchioni M M, et al. Brain MRI abnormalities in schizophrenia: Same genes or same environment? Psychol Med, 2005, 35: 1399-1409

11 Hall M H, Rijsdijk F, Picchioni M, et al. Substantial shared genetic influences on schizophrenia and event-related potentials. Am J Psychiatry, 2007, 164: 804-812

12 Nash M W, Huezo-Diaz P, Williamson R J, et al. Genome-wide linkage analysis of a composite index of neuroticism and moodrelated scales in extreme selected sibships. Hum Mol Genet, 2004, 13: 2173-2182

13 Purcell S, Cherny S S, Hewitt J K, et al. Optimal sibship selection for genotyping in quantitative trait locus linkage analysis. Hum Hered, 2001, 52: 1-13

14 Sham P C, Purcell S, Cherny S S, et al. Powerful regression-based quantitative-trait linkage analysis of general pedigrees. Am J Hum Genet, 2002, 71: 238-253

15 Potkin S G, Turner J A, Guffanti G, et al. A genome-wide association study of schizophrenia using brain activation as a quantitative phenotype. Schizophr Bull, 2009, 35: 96-108

16 Aberg K, Adkins D E, Liu Y, et al. Genome-wide association study of antipsychotic-induced QTc interval prolongation. Pharmacogenomics J, 2010, doi: $10.1038 /$ tpj.2010.76

17 Djurovic S, Gustafsson O, Mattingsdal M, et al. A genome-wide association study of bipolar disorder in Norwegian individuals, followed by replication in Icelandic sample. J Affect Disord, 2010, 126: $312-316$

18 Chen X, Lee G, Maher B S, et al. GWA study data mining and independent replication identify cardiomyopathy-associated 5 (CMYA5) as a risk gene for schizophrenia. Mol Psychiatry, 2010, doi: 10.1038/mp.2010.96

19 Need A C, Ge D, Weale M E, et al. A genome-wide investigation of SNPs and CNVs in schizophrenia. PLoS Genet, 2009, 5: e1000373

20 Purcell S M, Wray N R, Stone J L, et al. Common polygenic variation contributes to risk of schizophrenia and bipolar disorder. Nature, 2009, 460: 748-752

21 Abecasis G R, Cherny S S, Cookson W O, et al. Merlin-rapid analysis of dense genetic maps using sparse gene flow trees. Nat Genet, 2002, 30: 97-101

22 Abecasis G R, Cardon L R, Cookson W O. A general test of association for quantitative traits in nuclear families. Am J Hum Genet, 2000, 66: 279-292

23 Abecasis G R, Cookson W O, Cardon L R. Pedigree tests of transmission disequilibrium. Eur J Hum Genet, 2000, 8: 545-551

24 Purcell S, Neale B, Todd-Brown K, et al. PLINK: A tool set for whole-genome association and population-based linkage analyses. Am J Hum Genet, 2007, 81: 559-575

25 Allison D B, Thiel B, St Jean P, et al. Multiple phenotype modeling in gene-mapping studies of quantitative traits: Power advantages. Am J Hum Genet, 1998, 63: 1190-1201

26 Ferreira M A, Purcell S M. A multivariate test of association. Bioinformatics, 2009, 25: 132-133

27 Ferreira M A, Visscher P M, Martin N G, et al. A simple method to localise pleiotropic susceptibility loci using univariate linkage analyses of correlated traits. Eur J Hum Genet, 2006, 14: 953-962

28 Marlow A J, Fisher S E, Francks C, et al. Use of multivariate linkage analysis for dissection of a complex cognitive trait. Am J Hum Genet, 2003, 72: 561-570

29 Hall M H, Smoller J W. A new role for endophenotypes in the GWAS era: Functional characterization of risk variants. Harv Rev Psychiatry, 2010, 18: 67-74

30 Braff D L, Freedman R, Schork N J, et al. Deconstructing schizophrenia: An overview of the use of endophenotypes in order to understand a complex disorder. Schizophr Bull, 2007, 33: 21-32

31 Kas M J, Gelegen C, Schalkwyk L C, et al. Interspecies comparisons of functional genetic variations and their implications in neuropsychiatry. Am J Med Genet B Neuropsychiatr Genet, 2009, 150B: 309-317

32 Meyer-Lindenberg A, Weinberger D R. Intermediate phenotypes and genetic mechanisms of psychiatric disorders. Nat Rev Neurosci, 2006, 7: 818-827

33 Ferreira M A, O'Donovan M C, Meng Y A, et al. Collaborative genome-wide association analysis supports a role for ANK3 and CACNA1C in bipolar disorder. Nat Genet, 2008, 40: 1056-1058

34 Green E K, Grozeva D, Jones I, et al. The bipolar disorder risk allele at CACNA1C also confers risk of recurrent major depression and of schizophrenia. Mol Psychiatry, 2010, 15: 1016-1022

35 Bigos K L, Mattay V S, Callicott J H, et al. Genetic variation in CACNA1C affects brain circuitries related to mental illness. Arch Gen Psychiatry, 2010, 67: 939-945

36 Wessa M, Linke J, Witt S H, et al. The CACNA1C risk variant for bipolar disorder influences limbic activity. Mol Psychiatry, 2010, 15: 1126-1127

37 Dao D T, Mahon P B, Cai X, et al. Mood disorder susceptibility gene CACNAIC modifies mood-related behaviors in mice and interacts with sex to influence behavior in mice and diagnosis in humans. Biol Psychiatry, 2010, 68: 801-810

Open Access This article is distributed under the terms of the Creative Commons Attribution License which permits any use, distribution, and reproduction in any medium, provided the original author(s) and source are credited. 